\title{
Tecnologías educativas, habilidades sociales y la toma de decisiones en estudiantes universitarios
}

\section{Educational Technologies, Social Skills and Decision Making in University Students}

\author{
Arturo Eduardo Melgar Begazo* \\ Universidad César Vallejo, Lima, Perú \\ ORCID: https://orcid.org/0000-0002-1150-1519 \\ Willian Sebastián Flores Sotelo \\ Universidad Nacional Federico Villarreal, Lima, Perú \\ ORCID: https://orcid.org/0000-0003-3505-0676 \\ José Antonio Arévalo Tuesta \\ Universidad Nacional Federico Villarreal, Lima, Perú \\ ORCID: https://orcid.org/0000-0003-0341-7234 \\ Pedro Juan Antón de los Santos \\ Universidad Nacional Federico Villarreal, Lima, Perú \\ ORCID: https://orcid.org/0000-0003-0137-703X
}

Recibido 05-10-18 Revisado 20-11-18 Aprobado 25-03-19 En línea 02-04-19

*Correspondencia

Email: amelgar@ucv.edu.pe
Citar como:

Melgar, A., Flores, W., Arévalo, J. \& Antón, P. (2019). Tecnologías educativas, habilidades sociales y la toma de decisiones en estudiantes universitarios. Propósitos y Representaciones, 7(2), 440-456. doi: http://dx.doi.org/10.20511/pyr2019.v7n2.305 


\section{Resumen}

La presente investigación tuvo como objetivo determinar la relación que existe entre las tecnologías educativas, habilidades sociales y la toma de decisiones en estudiantes universitarios de la facultad de Ingeniería Industrial de una universidad privada de Lima. Este estudio fue de tipo básica, diseño no experimental-transversal. La población estuvo constituida por 80 alumnos. Se utilizó la técnica de la encuesta y su instrumento el cuestionario, con preguntas tipo escala de likert. Los resultados permitieron determinar que existe una relación significativa entre las tecnologías educativas, habilidades sociales y la toma de decisiones, con una significancia menor a .05 .

Palabras clave: Tecnologías educativas; Habilidades sociales; Toma de decisiones; Estudiantes universitarios; Universidad.

\section{Summary}

The objective of this research was to determine the relationship between educational technologies, social skills and decision making in university students of the Industrial Engineering School from a private university in Lima. This study was basic, its design was non-experimental and crosssectional. The population was composed of 80 students. The survey was used as a technique and the questionnaire was used as its instrument. The questionnaire had Likert Scale questions. The results allowed determining that there is a significant relationship between educational technologies, social skills and decision making, with a significance lower than .05 .

Keywords: Educational Technologies; Social Skills; Decision Making; University Students; University.

\section{Introducción}

Desde los años 70 se ha afirmado la importancia de la Tecnología Educativa. La Agencia Norteamericana para el Desarrollo Internacional (1972) la definió como una manera sistemática de perfeccionar el proceso de aprendizaje e instrucción, a través de investigaciones, con el fin de obtener resultados más efectivos. La Unesco, en 1978, estableció que la Tecnología Educativa se entiende como "[...] la aplicación de un enfoque científico y sistemático, con la información concomitante al mejoramiento de la educación, en sus variantes manifestadas y niveles diversos". La misma entidad, en 1984, definió el mismo término como "[...] el modo sistemático de concebir, aplicar y evaluar el conjunto de procesos de enseñanza y aprendizaje teniendo en cuenta a la vez los recursos técnicos y humanos y las interacciones entre ellos, como forma de obtener una más efectiva educación" (Area, 2009, p. 10).

Por otro lado, las habilidades sociales han sido definidas como "un conjunto de habilidades que permiten organizar cogniciones y conductas de manera eficiente, en la dirección del logro de metas interpersonales y sociales, que se realizan de un modo culturalmente aceptable" (Ladd \& Mize, 1983: Migueláñez, \& Conde, 2011). Investigaciones actuales en la temática han permitido incluir nuevas variables en este constructo, tales como la capacidad para prestar atención, la habilidad para trabajar en equipo, la capacidad para organizarse y para pedir ayuda a otros en caso de necesidad (Jacob, 2002, p. 589).

Adicionalmente, en el Informe del Perfil del Egresado Escuela de Ingeniería Industrial de la Universidad César Vallejo - 2015, se encontró el reporte del nivel bajo en la competencia 'Toma decisiones en base a información validada' de los egresados de esta escuela.

En relación al contexto de la presente investigación, Rivera (2009) presentó la utilidad que muestran las WebQuest, como un recurso didáctico en la educación superior, la necesidad de formación del profesorado para la integración curricular de las TIC y las WebQuest como 
herramienta didáctica para el desarrollo de competencias. Los datos pre y post-test de la investigación demostraron que las WebQuest, diseñadas por los docentes, facilitan el aprendizaje de los alumnos universitarios; los docentes capacitados las utilizan como herramienta didáctica en su práctica docente y finalmente, demostró que su uso es valorado satisfactoriamente por los estudiantes y aumenta el rendimiento en la asignatura.

Por su parte, Ruiz y Ruiz-Tapia (2010) en su investigación efectuó un análisis sobre el uso de un simulador de negocios como herramienta de aprendizaje para desarrollar la capacidad de toma de decisiones y trabajo en equipo en estudiantes de Contaduría y Administración de la Universidad Autónoma del Estado de México. Concluyó que la herramienta permite a los estudiantes enfrentar situaciones similares a las que enfrenta en una organización y debe aprender a resolver. De manera que cuando estas situaciones lleguen puedan contar con elementos de referencia para la toma de decisiones, con el desarrollo de creatividad, estrategias de pensamiento, observación y análisis.

\section{Tecnologías Educativas.}

Martínez (1996, citado en Cabero, 1996) identificó por nuevas tecnologías a todos aquellos medios de comunicación y tratamiento de la información, los cuales van surgiendo de la unión de los avances del desarrollo de la tecnología electrónica y las herramientas conceptuales (p. 53).

Además, señaló que en oposición a las tecnologías audiovisuales e informáticas que anteriormente se han acercado al mundo de la formación y el aprendizaje (como el cine, la radio, la enseñanza programada, la televisión, o el vídeo), respecto a las cuales podemos afirmar que no se han mostrado como opositoras al sistema educativo tradicional apoyado normalmente en la enseñanza en el aula, regidas por las variables del espacio y el tiempo; los nuevos desarrollos que se están presentando apoyados en las nuevas tecnologías de la información y comunicación, se muestran como verdaderas opositoras a la misma. Esto, al fomentar la interacción y el aprendizaje fuera de las barreras usuales del espacio y el tiempo, produciéndose en un no lugar denominado ciberespacio (p. 56). Asimismo, Salinas (citado en Cabero, 1998) indicó que la digitalización y los nuevos soportes electrónicos están dando lugar a nuevas formas de almacenar y presentar la información. Tutoriales multimedia, bases de datos en línea, bibliotecas electrónicas e hipertextos distribuidos responden a nuevas maneras de presentar y acceder al conocimiento. Dichas herramientas superan, en determinados contextos, las formas tradicionales de la explicación oral, la pizarra, los apuntes y el manual. No es necesario explicar las bondades de las simulaciones de procesos, la representación gráfica, la integración de texto, imagen y sonido o la navegación hipertexto. En el futuro, este soporte será utilizado de modo creciente en todos los niveles educativos (p. 33).

\section{Habilidades Sociales.}

Según Caballo (2007), las habilidades sociales:

Son un conjunto de conductas emitidas por el individuo en un contexto interpersonal que expresa sus sentimientos, actitudes, deseos, opiniones o derechos de un modo adecuado a la situación, respetando esas conductas en los demás y que, generalmente resuelve los problemas inmediatos de la situación mientras minimiza la probabilidad de futuros problemas (p. 6).

Tradicionalmente, las habilidades sociales han sido definidas como un conjunto de habilidades que permiten organizar cogniciones y conductas de manera eficiente. Todo esto, con la dirección del logro de metas interpersonales y sociales, que se realizan de un modo culturalmente aceptable (Ladd \& Mize, 1983; Organista, Silva, Salas, \& Lavigne, 2016; Fernández, 2017). Actualmente, la investigación en la temática ha permitido incluir nuevas variables en este constructo; tales como la capacidad para prestar atención, la habilidad para 
trabajar en equipo, la capacidad para organizarse y para pedir ayuda a otros en caso de necesidad (Jacob, 2002). Para Kelly (2002) son conductas aprendidas que utilizan las personas en situaciones interpersonales, para obtener o mantener reforzamiento del ambiente.

\section{Toma de Decisiones.}

Según Betancur (2016), tomar decisiones siempre inicia por elegir entre decidir o no decidir. Al decidir se está asumiendo el compromiso con los resultados que se quieren alcanzar, mientras que al no decidir se demuestra estar interesados en que las cosas sucedan, pero sin hacer nada para lograr los resultados deseados. Lo que lleva a una u otra cosa, son los modelos mentales y es por ello que se debe analizar los procesos de toma de decisiones a partir de estos.

Normalmente, se decide para alcanzar el éxito y se eluden las decisiones cuando se desea evitar el fracaso. De acuerdo a Sánchez (2014), la decisión institucional desencadena un conjunto específico de actividades que han ido concitando, progresiva y gradualmente, la atención de científicos y especialistas. Decidir es mucho más que el ejercicio personal de la libertad. La decisión es, en efecto, un acto complejo que pertenece a un proceso integrado por una serie de operaciones diversas, caracterizadas por su dimensión política. El líder institucional, al actuar como tal, pone en juego la dimensión social y práctica de la decisión (Minnaard, Servetto, Lobo Mirassón, \& Pascal, 2015; de Pablos Pons, 2018).

\section{Método}

La investigación fue de tipo básica, de naturaleza descriptiva y comparativa, orientada a la búsqueda de la relación entre las tecnologías educativas, habilidades sociales y la toma de decisiones en alumnos. Según Hernández, Fernández y Baptista (2014) es de tipo básica ya que contribuye al conocimiento científico. Al respecto se encontró que "dentro del enfoque cuantitativa, la calidad de una investigación se encuentra relacionada con el grado en que se aplique el diseño tal como fue preconcebido" (p. 136).

Los estudios descriptivos "buscan especificar las propiedades y los perfiles de personas, grupos, comunidades procesos, objetos o cualquier otro fenómeno que se someta a un análisis" (Hernández, Fernández \& Baptista, 2014, p. 80). Los estudios comparativos tienen "como propósito comparar la relación o grado de asociación que exista entre dos o más conceptos, categorías o verbales en un contexto en particular" (p. 81).

El diseño del presente trabajo fue no experimental, de corte transversal y descriptivo comparativo, ya que no se manipuló ni se sometió a prueba las variables en estudio. No experimental es "la investigación que se realiza sin manipular deliberadamente variables. Es decir, se trata de estudios donde no hacemos variar en forma intencional las variables independientes para ver su efecto sobre otras variables" (Hernández et al., 2014, p. 149).

Es descriptivo comparativo porque este diseño "permite recolectar información relevante en varias muestras con respecto a un mismo fenómeno o aspecto de interés y luego caracterizar este fenómeno en base a la comparación de los datos recogidos" (Sierra, 2007, p. 3).

Es transversal, ya que su propósito es "describir variables y analizar su incidencia e interrelación en un momento dado. Es como tomar una fotografía de algo que sucede" (Hernández et al., 2014, p. 151).

\section{Población y muestra}

La población está constituida por los estudiantes del quinto año de la Escuela de Ingeniería Industrial de una universidad privada de Lima. La muestra fue censal, por lo que se estudió al total de la población, correspondiente a 80 alumnos de la escuela profesional en 
mención.

\section{Técnica.}

La técnica utilizada fue la encuesta. "La encuesta es un instrumento de la investigación de mercados que consiste en obtener información de las personas encuestadas mediante el uso de cuestionarios diseñados en forma previa para la obtención de información específica" (Hernández et al., 2014, p.1). El instrumento utilizado en la investigación fue el cuestionario. Se aplicaron tres cuestionarios, uno para cada variable en un mismo momento.

\section{Resultados}

De acuerdo al análisis estadístico, luego de la aplicación de los instrumentos se procedió a encontrar los siguientes resultados:

Tabla 1.

Relación entre tecnologías educativas y habilidades sociales.

\begin{tabular}{|c|c|c|c|c|c|c|c|c|c|c|c|c|}
\hline \multirow[t]{2}{*}{ Modelo } & \multirow[t]{2}{*}{$\mathrm{R}$} & \multirow{2}{*}{$\begin{array}{c}\mathrm{R} \\
\text { cuadrado }\end{array}$} & \multirow{2}{*}{$\begin{array}{c}\text { R cuadrado } \\
\text { corregida }\end{array}$} & \multirow{2}{*}{$\begin{array}{c}\text { Error típ. } \\
\text { de la } \\
\text { estimació }\end{array}$} & \multicolumn{8}{|c|}{ Estadísticos de cambio } \\
\hline & & & & & \multicolumn{2}{|c|}{$\begin{array}{c}\text { Cambio en } \mathrm{R} \\
\text { cuadrado }\end{array}$} & $\begin{array}{c}\text { Cambio } \\
\text { en F }\end{array}$ & gl1 & $\mathrm{gl} 2$ & \multicolumn{2}{|c|}{$\begin{array}{l}\text { Sig. Cambio } \\
\text { en } \mathrm{F}\end{array}$} & $\begin{array}{l}\text { Durbin- } \\
\text { Watson }\end{array}$ \\
\hline 1 & $.859^{\mathrm{a}}$ & .739 & .735 & .366 & & 39 & 220.371 & $1^{\mathrm{a}}$ & 78 & .00 & & 2.014 \\
\hline \multicolumn{13}{|c|}{ Coeficientes $^{\mathrm{a}}$} \\
\hline \multirow[t]{2}{*}{ Modelo } & & & & \multicolumn{2}{|c|}{$\begin{array}{l}\text { Coeficientes no } \\
\text { estandarizados }\end{array}$} & \multicolumn{2}{|c|}{$\begin{array}{c}\text { Coeficientes } \\
\text { tipificados }\end{array}$} & $\mathrm{t}$ & Sig. & \multicolumn{3}{|c|}{ Correlaciones } \\
\hline & & & & B $\quad$ Err & or típ. & $\mathrm{Be}$ & & & & $\begin{array}{c}\text { Orden } \\
\text { cero }\end{array}$ & $\begin{array}{l}\text { Parci } \\
\text { al }\end{array}$ & $\begin{array}{c}\text { Semi } \\
\text { parcial }\end{array}$ \\
\hline (Con & stante) & & & 215 & 145 & & & 1.487 & .141 & & & \\
\hline $\begin{array}{l}\text { TEC } \\
\text { EDU } \\
\text { HAB } \\
(\mathrm{x} 2)\end{array}$ & $\begin{array}{l}\text { NOLOC } \\
\text { CATIV } \\
\text { ILIDA } \\
\text { (agrupa }\end{array}$ & $\begin{array}{l}\text { JÍAS } \\
\text { AS (x1) - } \\
\text { DES SOCI } \\
\text { do) }\end{array}$ & ALES & 891 & 060 & .85 & & 14.845 & .000 & .859 & .859 & .859 \\
\hline
\end{tabular}

Dado que $\mathrm{R}=0.859$, el $85.90 \%$ de las variables tecnologías educativas y habilidades sociales correlacionan a la variable toma de decisiones; el coeficiente tipificado beta 0.859 indica el porcentaje que afectan la variable que sería $85.90 \%$. El valor sig .000 menor a significancia .05; por lo tanto, existe una relación significativa entre las tecnologías educativas, habilidades sociales y la toma de decisiones, con una alta correlación (0.859).

Tabla 2.

Relación entre tecnologías educativas y toma de decisiones.

\begin{tabular}{|c|c|c|c|c|c|c|c|c|c|c|c|c|}
\hline \multirow{2}{*}{\multicolumn{2}{|c|}{ Modelo }} & \multirow[t]{2}{*}{$\mathrm{R}$} & \multirow{2}{*}{$\begin{array}{c}\mathrm{R} \\
\text { cuadrado }\end{array}$} & \multirow{2}{*}{$\begin{array}{c}\mathrm{R} \text { cuadrado } \\
\text { corregida }\end{array}$} & \multirow{2}{*}{$\begin{array}{l}\text { Error típ. } \\
\text { de la } \\
\text { estimación }\end{array}$} & \multicolumn{7}{|c|}{ Estadísticos de cambio } \\
\hline & & & & & & $\begin{array}{c}\text { Cambio en R } \\
\text { cuadrado }\end{array}$ & $\begin{array}{c}\text { Cambio } \\
\text { en F }\end{array}$ & gl1 & gl2 & \multicolumn{2}{|c|}{$\begin{array}{l}\text { Sig. Cambio } \\
\text { en F }\end{array}$} & $\begin{array}{l}\text { Durbin- } \\
\text { Watson }\end{array}$ \\
\hline & 1 & $.733^{\mathrm{a}}$ & .537 & .531 & .487 & .537 & 90.336 & $1^{\mathrm{a}}$ & 78 & .00 & & 1.576 \\
\hline \multicolumn{13}{|c|}{ Coeficientes $^{\mathrm{a}}$} \\
\hline \multirow{2}{*}{\multicolumn{4}{|c|}{ Modelo }} & & \multicolumn{2}{|c|}{$\begin{array}{l}\text { Coeficientes no } \\
\text { estandarizados }\end{array}$} & $\begin{array}{c}\text { Coeficientes } \\
\text { tipificados }\end{array}$ & $\mathrm{t}$ & Sig. & \multicolumn{3}{|c|}{ Correlaciones } \\
\hline & & & & & Err & or típ. & & & & $\begin{array}{c}\text { Orden } \\
\text { cero }\end{array}$ & $\begin{array}{c}\text { Parci } \\
\text { al }\end{array}$ & $\begin{array}{c}\text { Semi } \\
\text { parcial }\end{array}$ \\
\hline \multirow[b]{2}{*}{1} & (Con & stante) & & & 682 & 176 & & 3.871 & .000 & & & \\
\hline & $\begin{array}{l}\text { TEC } \\
\text { EDU } \\
\text { (agru }\end{array}$ & $\begin{array}{l}\text { NOLO } \\
\text { CATIV } \\
\text { pado) }\end{array}$ & $\begin{array}{l}\text { IÍAS } \\
\text { AS (x1) }\end{array}$ & & 689 & 072 & 33 & 9.505 & .000 & .733 & .733 & .733 \\
\hline
\end{tabular}


Dado que $\mathrm{R}=0.733$ el $73,30 \%$ de la variable tecnologías educativas correlaciona a la variable toma de decisiones. El valor sig .000 menor a significancia .05; por lo tanto, existe una relación significativa entre las tecnologías educativas y la toma de decisiones, con una alta correlación (0.733).

Tabla 3.

Relación entre el simulador de procesos, blended learning y la toma de decisiones.

\begin{tabular}{|c|c|c|c|c|c|c|c|c|c|c|c|c|}
\hline \multirow[t]{2}{*}{ Modelo } & \multirow[t]{2}{*}{$\mathrm{R}$} & \multirow{2}{*}{$\begin{array}{c}\mathrm{R} \\
\text { cuadrado }\end{array}$} & \multirow{2}{*}{$\begin{array}{l}\mathrm{R} \text { cuadrado } \\
\text { corregida }\end{array}$} & \multirow{2}{*}{$\begin{array}{c}\text { Error típ. } \\
\text { de la } \\
\text { estimación }\end{array}$} & \multicolumn{8}{|c|}{ Estadísticos de cambio } \\
\hline & & & & & \multicolumn{2}{|c|}{$\begin{array}{l}\text { Cambio en } \mathrm{R} \\
\text { cuadrado }\end{array}$} & $\begin{array}{c}\text { Cambio } \\
\text { en F }\end{array}$ & gl1 & $\mathrm{gl} 2$ & \multicolumn{2}{|c|}{$\begin{array}{l}\text { Sig. Cambio } \\
\text { en F }\end{array}$} & $\begin{array}{l}\text { Durbin- } \\
\text { Watson }\end{array}$ \\
\hline 1 & $.769^{\mathrm{a}}$ & .591 & .581 & .460 & & 591 & 55.744 & $2^{\mathrm{a}}$ & 77 & .00 & & 1.635 \\
\hline \multicolumn{13}{|c|}{ Coeficientes $^{\mathrm{a}}$} \\
\hline \multirow[t]{2}{*}{ Modelo } & & & & \multicolumn{2}{|c|}{$\begin{array}{l}\text { Coeficientes no } \\
\text { estandarizados }\end{array}$} & \multicolumn{2}{|c|}{$\begin{array}{l}\text { Coeficientes } \\
\text { tipificados }\end{array}$} & $\mathrm{t}$ & Sig. & \multicolumn{3}{|c|}{ Correlaciones } \\
\hline & & & & Err & or típ. & $\mathrm{Be}$ & & & & $\begin{array}{c}\text { Orden } \\
\text { cero }\end{array}$ & $\begin{array}{c}\text { Parci } \\
\text { al }\end{array}$ & $\begin{array}{c}\text { Semi } \\
\text { parcial }\end{array}$ \\
\hline \multicolumn{2}{|c|}{ (Constante) } & & & 447 & 226 & & & 1.981 & .051 & & & \\
\hline \multicolumn{3}{|c|}{$\begin{array}{l}\text { Blended learning }(\mathrm{d} 1 \mathrm{x} 1) \\
\text { (agrupado) }\end{array}$} & & 583 & 103 & \multicolumn{2}{|c|}{.629} & 5.650 & .000 & .761 & .541 & .412 \\
\hline \multicolumn{3}{|c|}{$\begin{array}{l}\text { Simulador de procesos } \\
(\mathrm{d} 2 \times 1) \text { (agrupado) }\end{array}$} & & 209 & 133 & \multicolumn{2}{|c|}{.175} & 1.570 & .120 & .650 & .176 & .114 \\
\hline
\end{tabular}

Dado que $\mathrm{R}=0.769$ el $76.90 \%$ de las dimensiones simulador de procesos, blended learning correlacionan a la variable toma de decisiones; se puede interpretar con los coeficientes tipificados beta 0.629 el blended learning afecta un $62,90 \%$ a la variable. Valor sig .000 menor a significancia .05 (blended learning), sig $0.120>.05$ (simulador de procesos); por lo tanto, no existe una relación significativa entre el simulador de procesos, blended learning y la toma de decisiones.

Tabla 4.

Relación entre las habilidades sociales y la toma de decisiones.

\begin{tabular}{|c|c|c|c|c|c|c|c|c|c|c|c|c|}
\hline \multirow[t]{2}{*}{ Modelo } & \multirow[t]{2}{*}{$\mathrm{R}$} & \multirow{2}{*}{$\begin{array}{c}\mathrm{R} \\
\text { cuadrado }\end{array}$} & \multirow{2}{*}{$\begin{array}{l}\mathrm{R} \text { cuadrado } \\
\text { corregida }\end{array}$} & \multirow{2}{*}{$\begin{array}{l}\text { Error típ. } \\
\text { de la } \\
\text { estimación }\end{array}$} & \multicolumn{8}{|c|}{ Estadísticos de cambio } \\
\hline & & & & & \multicolumn{2}{|c|}{$\begin{array}{c}\text { Cambio en R } \\
\text { cuadrado }\end{array}$} & $\begin{array}{l}\text { Cambio } \\
\text { en F }\end{array}$ & gl1 & $\mathrm{gl2}$ & \multicolumn{2}{|c|}{$\begin{array}{l}\text { Sig. Cambio } \\
\text { en F }\end{array}$} & $\begin{array}{l}\text { Durbin- } \\
\text { Watson }\end{array}$ \\
\hline 1 & $.855^{\mathrm{a}}$ & .732 & .728 & .371 & & 32 & 212.691 & $1^{\mathrm{a}}$ & 78 & .00 & & 2.102 \\
\hline \multicolumn{13}{|c|}{ Coeficientes $^{\mathrm{a}}$} \\
\hline \multirow[t]{2}{*}{ Modelo } & & & & \multicolumn{2}{|c|}{$\begin{array}{l}\text { Coeficientes no } \\
\text { estandarizados }\end{array}$} & \multicolumn{2}{|c|}{$\begin{array}{l}\text { Coeficientes } \\
\text { tipificados }\end{array}$} & $\mathrm{t}$ & Sig. & \multicolumn{3}{|c|}{ Correlaciones } \\
\hline & & & & Err & or típ. & \multicolumn{2}{|c|}{ Beta } & & & $\begin{array}{c}\text { Orden } \\
\text { cero }\end{array}$ & $\begin{array}{c}\text { Parci } \\
\text { al }\end{array}$ & $\begin{array}{cc}\text { i } & \text { Semi } \\
\text { parcial }\end{array}$ \\
\hline \multicolumn{2}{|c|}{ (Constante) } & & & 325 & 140 & & & 2.321 & .023 & & & \\
\hline \multicolumn{4}{|c|}{$\begin{array}{l}\text { HABILIDADES SOCIALES } \\
\text { (x2) (agrupado) }\end{array}$} & 839 & 058 & \multicolumn{2}{|c|}{,855 } & 14.584 & .000 & .855 & .855 & .855 \\
\hline
\end{tabular}

Dado que $\mathrm{R}=0.855$ el $85.50 \%$ de la variable habilidades sociales correlaciona a la variable toma de decisiones; asimismo, el valor sig .000 menor a significancia .05; por lo tanto, existe una relación significativa entre las habilidades sociales y la toma de decisiones, con una alta correlación (0.855). 
Tabla 5.

Relación entre primeras habilidades, habilidades avanzadas, manejo sentimientos, habilidades alternativas, habilidad frente al estrés, habilidad de planificación y la toma de decisiones

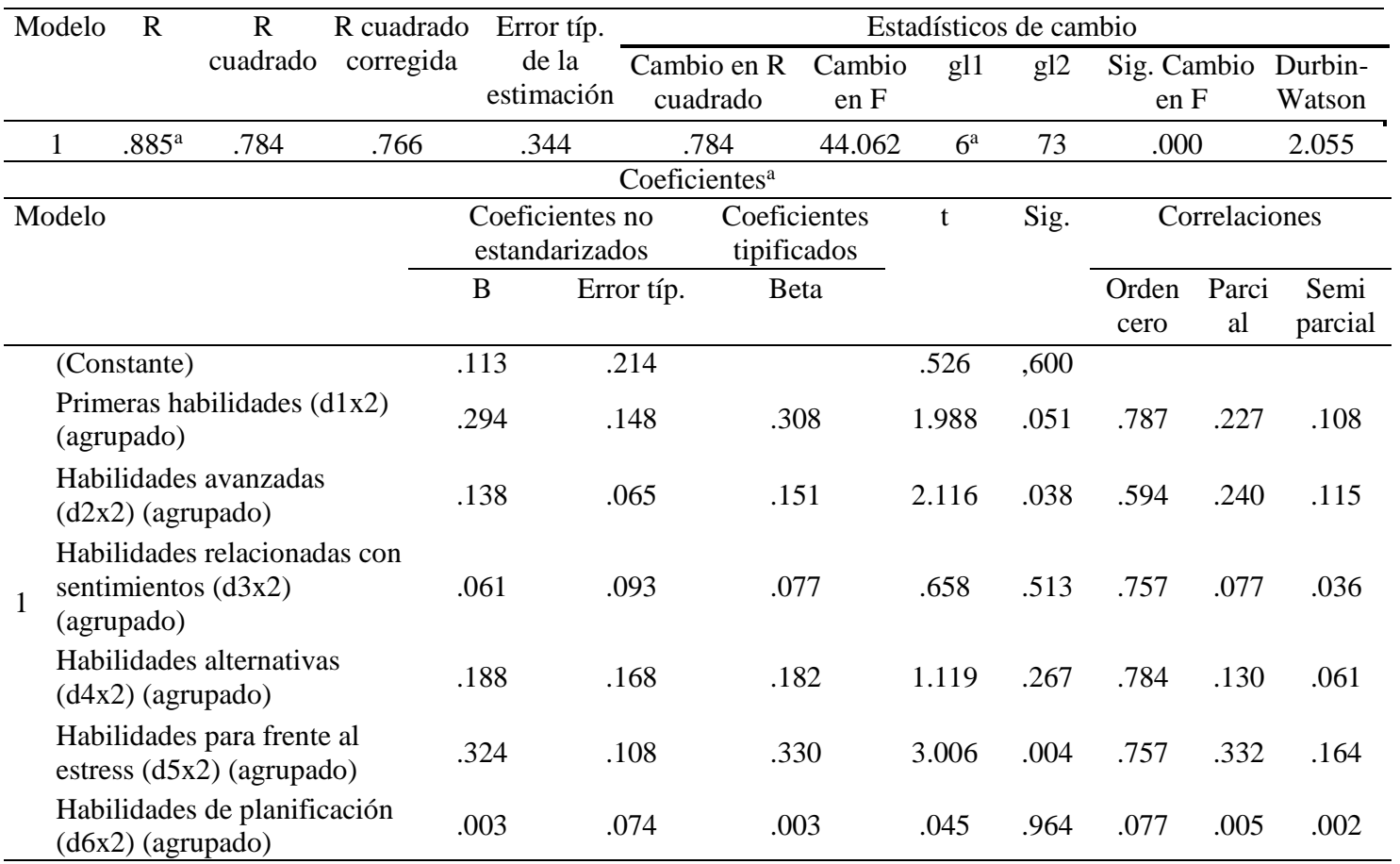

Dado que $\mathrm{R}=0.885$ el $88.50 \%$ de las dimensiones primeras habilidades, habilidades avanzadas, manejo sentimientos, habilidades alternativas, habilidad frente al estrés, habilidad de planificación correlacionan a la variable toma de decisiones; los valores sig .051; $0.513 ; 0.267$ y 0.964 no son menores a significancia .05 solo .038 ; .004 son menores a .05. Entonces, no existe una relación significativa entre primeras habilidades, habilidades avanzadas, manejo sentimientos, habilidades alternativas, habilidad frente al estrés, habilidad de planificación y la toma de decisiones.

\section{Discusión}

Para la variable tecnologías educativas, la investigación de Rivera (2009) señaló que las WebQuest diseñadas por profesores facilitan el aprendizaje de los alumnos universitarios. Así como demuestra que su uso en la enseñanza universitaria es valorado satisfactoriamente por los estudiantes y aumenta el rendimiento en las asignaturas. En el mismo sentido, en la presente investigación se encontró que existe una relación significativa entre las tecnologías educativas y la toma de decisiones en alumnos del quinto año de Ingeniería Industrial de una universidad privada de Lima, con una correlación alta, analizando las tecnologías como blended learning y simuladores de procesos. Se demostró la importancia en la utilización de las nuevas tecnologías educativas para mejorar la competencia toma de decisiones en los estudiantes del último año de la Escuela de Ingeniería Industrial.

Igualmente, para la variable tecnologías educativas, Ruiz (2010) efectuó el análisis del uso del simulador de negocios Risky Business como herramienta de aprendizaje para desarrollar la capacidad de toma de decisiones y trabajo en equipo a través de la integración de los conceptos administrativos de estudiantes universitarios de Contabilidad y Administración. En el mismo sentido, en la presente investigación se encontró que existe una relación significativa entre las tecnologías educativas y la toma de decisiones en alumnos de quinto año de Ingeniería Industrial, por lo cual se recomienda hacer un nuevo estudio cuasi-experimental para ver la influencia de 
las herramientas de simulación de procesos en la toma de decisiones de los estudiantes.

En cuanto a las habilidades sociales, en el caso de García (2005) encontró una correlación significativa positiva entre habilidades sociales y clima social en la familia. Sin embargo, encontró que no existe una correlación significativa entre habilidades sociales y clima social en la familia con el rendimiento académico. En contraste con ello, en la presente investigación se encontró que existe una relación significativa entre las habilidades sociales y toma de decisiones en alumnos del quinto año de Ingeniería Industrial con una correlación muy alta, lo cual ha permitido encontrar la importancia de las habilidades sociales en la toma de decisiones de estos estudiantes de quinto año.

\section{Referencias}

Area, M. (2010). Introducción a la tecnología educativa. DIM: Didáctica, Innovación y Multimedia, 19, 1-78. Recuperado de: https://www.raco.cat/index.php/DIM/article/viewFile/306306/396214

Betancur, J. R. (2016). Toma de decisiones: obtener el éxito [degerencia.com]. Recuperado de http://www.degerencia.com/articulo/toma_de_decisiones_obtener_el_exito

Caballo, V. (2007). Manual de evaluación y entrenamiento de las habilidades sociales. (7ma. ed.). Madrid: Siglo XXI.

Cabero, J. (1996). El ciberespacio: el no lugar como lugar educativo. In Salinas, J.; otros (Coords.). Redes de Comunicación, redes de aprendizaje. Actas del Congreso Edutec. Universidad de las Islas Baleares. Palma.

de Pablos Pons, J. (2018). Las tecnologías digitales y su impacto en la Universidad. Las nuevas mediaciones. RIED. Revista Iberoamericana de Educación a Distancia, 21(2), 83-95. Recuperado de: http://revistas.uned.es/index.php/ried/article/view/20733

Fernández, C. Q. (2017). Estrategias para la innovación e implementación de las Tecnologías de la Información y la Comunicación en la práctica educativa: una respuesta a las nuevas demandas de la sociedad del siglo XXI. Posgrado y Sociedad. Revista Electrónica del Sistema de Estudios de Posgrado, 11(1), 22-32. Doi: http://dx.doi.org/10.22458/rpys.v11i1.392

García, C. (2005). Habilidades sociales, clima social familiar y rendimiento académico en estudiantes universitarios. Liberabit, 11(11), 63-74.

Hernández, R., \& Fernández, C. \& Baptista, M. (2014) Metodología de la Investigación. México. Editorial Mc. Graw Hill Interamericana Editores, SA.

Jacob, B. (2002). Where the boys aren't: non-cognitive skills, returns to school and the gender gap in higher education. Economics of Education Review, 21, 589 - 598. Doi: https://doi.org/10.1016/S0272-7757(01)00051-6

Kelly, J. A. (2002). Entrenamiento de las Habilidades Sociales (7ma ed.). Bilbao: Desclée de Brower.

Ladd, G. W., \& Mize, J. (1983). A cognitive-social learning model of social-skill training. Psychological review, 90(2), 127. Doi: http://dx.doi.org/10.1037/0033-295X.90.2.127

Minnaard, C. L., Servetto, D., Lobo Mirassón, U., \& Pascal, G. (2015). La información y la tecnología para la toma de decisiones: Aplicación Data WareHouse en Instituciones Universitarias. In Workshop de Investigadores en Ciencias de la Computación.

Migueláñez, S. O., \& Conde, M. J. R. (2011). Perspectiva tecnológica de la evaluación educativa en la universidad. Teoría de la Educación. Revista Interuniversitaria, 23(1), 131-157. Recuperado de: https://gredos.usal.es/jspui/bitstream/10366/121619/1/Perspectiva_tecnologica_de_la_evaluacion. pdf

Organista, J., Silva, M. S., Salas, L. M., \& Lavigne, G. (2016). Estimación de las habilidades digitales con propósito educativo de estudiantes de dos universidades públicas mexicanas. Edutec. Revista Electrónica de Tecnología Educativa, 57. Recuperado de: https://doi.org/10.21556/edutec.2016.57.673

Rivera, Y. (2009). Evaluación de las WebQuest como herramientas didácticas en la Educación Superior. Enseñanza \& Teaching: Revista Interuniversitaria de Didáctica, 28(1), 139-155. Recuperado de http://revistas.usal.es/index.php/0212-5374/article/view/7485

Ruiz, S., Ruiz-Tapia, J. (2010). Estrategias competitivas en el aprendizaje con el uso de un simulador de negocios en alumnos de educación superior de la UAEMEX. RECAI Revista de Estudios en Contaduría, Administración e Informática, 2(1). Recuperado: https://recai.uaemex.mx/article/view/8952

Sánchez, R. (2014). Enseñar a investigar. Una didáctica nueva de la investigación en ciencias sociales y humanas. México, D. F.: Universidad Nacional Autónoma de México. 